IMPERIAL/TP/95-96/56

\title{
Nonperturbative calculation of symmetry breaking in quantum field theory
}

\author{
Carl M. Bender幽 \\ Blackett Laboratory, Imperial College, London SW7 2BZ, United Kingdom \\ Kimball A. Milton目 \\ Department of Physics and Astronomy, University of Oklahoma, Norman, OK 73019, USA
}

(June 27, 2021)

\begin{abstract}
A new version of the delta expansion is presented, which, unlike the conventional delta expansion, can be used to do nonperturbative calculations in a self-interacting scalar quantum field theory having broken symmetry. We calculate the expectation value of the scalar field to first order in $\delta$, where $\delta$ is a measure of the degree of nonlinearity in the interaction term.
\end{abstract}

Typeset using REVTEX 
In a private discussion with $\mathrm{D}$. Bessis we learned about the remarkable non-Hermitian Hamiltonian

$$
H=p^{2} / 2+i x^{3} .
$$

The eigenvalues of this Hamiltonian are all real and positive! (We have no proof of this property, but there is ample numerical evidence.) This Hamiltonian is intriguing because it suggests a novel way to apply the delta expansion to quantum field theories having a broken symmetry. In this paper we introduce our new version of the delta expansion and use it to calculate the (nonzero) value of $\langle\phi\rangle$ in a scalar quantum field theory [see Eq. (31)].

The delta expansion is a Taylor series in powers of $\delta$, where $\delta$ measures the degree of nonlinearity of an interaction term [1]. For a scalar quantum field theory, the conventional approach [2] has been to introduce the parameter $\delta$ into the Euclidean Lagrangian density by

$$
\mathcal{L}=(\nabla \phi)^{2} / 2+m^{2} \phi^{2} / 2+g\left(\phi^{2}\right)^{1+\delta} .
$$

The advantage of the delta expansion is that it is nonperturbative in the coupling constant $g$ and mass $m$ and has a nonzero radius of convergence. The conventional delta expansion has been used to study renormalization [0] , supersymmetry [4], local gauge invariance [5], stochastic quantization [6], and finite-temperature field theory [7].

A drawback of the Lagrangian density in Eq. (22) is that it becomes a $g|\phi|^{3}$ theory rather than a $g \phi^{3}$ theory when $\delta=\frac{1}{2}$. The $g|\phi|^{3}$ theory is symmetric under $\phi \rightarrow-\phi$ and cannot exhibit symmetry breaking. In general, the conventional delta expansion is unsuitable for studying theories with symmetry breaking.

In the past it was assumed that to satisfy the physical requirement that the Hamiltonian be bounded below for all $\delta$, the parameter $\delta$ should appear in the Lagrangian density (2) as the exponent of $\phi^{2}$ and not of $\phi$. This assumption appears to be even more reasonable if one expands the interaction term in Eq. (2) in powers of $\delta$ :

$$
g\left(\phi^{2}\right)^{1+\delta}=g \phi^{2} \sum_{k=0}^{\infty} \frac{\delta^{k}}{k !}\left(\log \phi^{2}\right)^{k} .
$$


One would think that the argument of the logarithm in Eq. (3) should be positive to avoid complex numbers.

However, $H$ in Eq. (1) describes a theory having a positive spectrum. This suggests a surprising new version of the delta expansion in which we replace Eq. (2) by

$$
\mathcal{L}=(\nabla \phi)^{2} / 2+m^{2} \phi^{2} / 2-g(i \phi)^{2+\delta}
$$

As with Eq. (2), $\delta=0$ gives free field theory. At $\delta=1$ we recover (when the dimension of space-time is one and the bare mass vanishes) the theory described by Eq. (11).

We believe that for $\operatorname{Re} \delta>-2$ the quantum-mechanical theory has a positive real spectrum. Here, we verify this to first order in $\delta$. The shift in the $n$th energy level of $H=p^{2} / 2-(i x)^{2+\delta}$ is the expectation value of $\delta x^{2} \log (i x)$ in the unperturbed harmonicoscillator basis $|n\rangle$ :

$$
\begin{aligned}
\Delta E_{n} & =\left\langle n\left|\delta x^{2} \log (i x)\right| n\right\rangle \\
& =\delta \int_{-\infty}^{\infty} d x \psi_{n}^{2}(x) x^{2} \log (i x) \\
& =\delta\left(\int_{-\infty}^{0}+\int_{0}^{\infty}\right) d x \psi_{n}^{2}(x) x^{2} \log (i x) \\
& =\delta \int_{0}^{\infty} d x \psi_{n}^{2}(x) x^{2}[\log (-i x)+\log (i x)] \\
& =2 \delta \int_{0}^{\infty} d x \psi_{n}^{2}(x) x^{2} \log (x) \\
& =\delta 2^{-5 / 2}\left[a_{n}-(2 n+1) \gamma-(5 n+5 / 2) \log 2\right] .
\end{aligned}
$$

The sequence $a_{n}=2,8,16,74 / 3, \ldots$ is given by

$$
a_{n}=4 n+4+4[n / 2]+(4 n+2) \sum_{k=0}^{[n / 2+1 / 2]} \frac{1}{2 k-1},
$$

where $[\alpha]$ is the greatest integer in $\alpha$. (In the above calculation we choose the branch of the logarithm to lie along the negative axis and choose the sheet of the Riemann surface for which $\log 1=0$.) Although the Hamiltonian is not self-adjoint, the $\mathrm{O}(\delta)$ shift in the energy levels is real! This property persists to all orders in $\delta$ [8].

We will now show that in any Euclidean dimension and for all $\delta>-2$ (apart from a special discrete set of negative values of $\delta$ ), Eq. (4) exhibits symmetry breaking. This result 
is especially interesting for the case $\delta=2$ because, although the resulting $\phi^{4}$ theory appears symmetric under $\phi \rightarrow-\phi$, symmetry breaking persists!

We begin by considering a massless zero-dimensional scalar quantum field theory, whose vacuum-persistence amplitude $Z$ and $N$-point Green's function $G_{N}$ are

$$
Z=\int_{-\infty}^{\infty} d x e^{(i x)^{2+\delta}}, \quad G_{N}=\frac{1}{Z} \int_{-\infty}^{\infty} d x e^{(i x)^{2+\delta}} x^{N}
$$

To evaluate $G_{N}$ we break the integral into two pieces:

$$
\begin{aligned}
Z G_{N} & =\int_{-\infty}^{0} d x e^{(i x)^{2+\delta}} x^{N}+\int_{0}^{\infty} d x e^{(i x)^{2+\delta}} x^{N} \\
& =2\left(\begin{array}{c}
\operatorname{Re}(\text { if } N \text { even }) \\
i \operatorname{Im}(\text { if } N \text { odd })
\end{array}\right) \int_{0}^{\infty} d x e^{(i x)^{2+\delta}} x^{N}
\end{aligned}
$$

This integral exists if $-1<\operatorname{Re} \delta<1$. We let $x^{2+\delta}=s$ and use standard Fresnel contour integral methods to get

$$
Z G_{N}=-(-i)^{N} \frac{2 \pi}{N+1} / \Gamma\left(-\frac{1+N}{2+\delta}\right)
$$

Using this result we calculate $G_{N}$ :

$$
G_{N}=\frac{(-i)^{N}}{N+1} \Gamma\left(-\frac{1}{2+\delta}\right) / \Gamma\left(-\frac{1+N}{2+\delta}\right)
$$

The nonvanishing of $G_{2 N+1}$ in Eq. (9) confirms that there is symmetry breaking.

Let us examine the case $\delta=2\left(\phi^{4}\right.$ theory) in depth. Since the one-point Green's function $G_{1}$ for this model is nonvanishing, the symmetry remains permanently broken as $\delta$ approaches 2. The Green's functions for this theory are $G_{N}=(-i)^{N} \Gamma(3 / 4) / \Gamma(3 / 4-N / 4)$. In the presence of an external source $J$, the vacuum functional $Z[J]$ for the theory has a convergent Taylor series in $J$ :

$$
Z[J]=Z[0] \sum_{N=0}^{\infty} \frac{J^{N} G_{N}}{N !}=\frac{\pi}{2} \sum_{N=0}^{\infty} \frac{J^{N}(-i)^{N}}{N ! \Gamma\left(\frac{3-N}{4}\right)}
$$

Note that $Z[J]$ obeys the third-order differential equation

$$
Z^{\prime \prime \prime}[J]+J Z[J] / 4=0 .
$$


This is the $D=0$ form of the functional Schwinger-Dyson equation for the theory. If $J$ is rotated by $e^{i \pi / 4}$, Eq. (11) becomes the differential equation satisfied by the vacuum functional $\tilde{Z}[J]$ for a conventional zero-dimensional $\phi^{4}$ theory in the presence of an external source:

$$
\tilde{Z}[J]=\int_{-\infty}^{\infty} d x e^{-x^{4}+J x}
$$

This model does not exhibit symmetry breaking.

The Schwinger-Dyson equation (11) does not specify the vacuum functional uniquely [9.10]. Indeed, there are four distinct solutions to the rotated version of Eq. (11):

$$
Z_{j}[J]=\int_{C_{j}} d x e^{-x^{4}+J x} \quad(j=1,2,3,4)
$$

Here, $C_{j}$ are contours in the complex- $x$ plane; $C_{1}$ joins $i \infty$ to $+\infty, C_{2}$ joins $+\infty$ to $-i \infty$, $C_{3}$ joins $-i \infty$ to $-\infty$, and $C_{4}$ joins $-\infty$ to $i \infty$. (The four solutions $Z_{j}[J]$ are not linearly independent; their sum is zero.) The vacuum functional $\tilde{Z}[J]$ in Eq. (12) is given by $Z_{4}[J]+$ $Z_{1}[J]$. The vacuum functional for the symmetry-broken theory in Eq. (10) is $e^{i \pi / 4} Z_{1}\left[e^{3 i \pi / 4} J\right]$. The symmetry-broken theory corresponding to $\delta=2$ in Eq. (6) is distinguished from the conventional unbroken theory in Eq. (12) in that the sign of the $x^{4}$ term is reversed (reflecting the factor of $e^{3 i \pi / 4}$ in the source term above).

Next, let us examine the analytic behavior of Eq. (6) as a function of complex $\delta$. The delta expansion of the one-point Green's function $G_{1}$, whose nonvanishing signals the presence of symmetry breaking, is

$$
\begin{gathered}
G_{1}=-i \sqrt{\pi}\left(\delta / 2+\delta^{2}(\gamma-2-2 \log 2) / 8-\delta^{3}\left[48+\pi^{2}\right.\right. \\
\left.\left.-6(\gamma-4-2 \log 2)^{2}\right] / 384+\ldots\right) .
\end{gathered}
$$

The radius of convergence of this expansion is 2 because there is one singularity in the $\delta$ plane at $\delta=-2$. [One might think (incorrectly) that as $\delta \rightarrow-1$ the integral for $Z$ in Eq. (6) would become a representation of a Dirac delta function, which is singular. However, it is not generally true that when an integral representation for a function ceases to exist, the 
function exhibits a singularity [11].] $G_{N}$ is analytic for $\operatorname{Re} \delta>-2$ because the integration path in Eq. (6) is an implicit function of $\delta$. Indeed, as delta ranges through real values, the paths of integration of the two integrals in the first line of Eq. (7) rotate in opposite directions [12. The path of integration of the first integral connects 0 and $\infty$ in the complex- $x$ plane along the straight line

$$
\text { path 1: } \quad \arg x=3 \pi / 2-\pi /(2+\delta) .
$$

The second integration path runs from 0 to $\infty$ along

$$
\text { path } 2: \quad \arg x=\pi /(2+\delta)-\pi / 2 \text {. }
$$

When $\delta=0$ (free field theory), path 1 connects $-\infty$ to 0 and path 2 connects 0 to $\infty$ along the real- $x$ axis. For this case $G_{N}$ is real and there is no symmetry breaking. As $\delta$ increases, path 1 rotates anticlockwise and path 2 rotates clockwise. The two paths slope downward at $45^{\circ}$ angles when $\delta=2$ ( $\phi^{4}$ field theory). As $\delta \rightarrow \infty$, both paths connect the origin to $-i \infty$. Since the paths overlap and the integrations are in opposite senses, the two integrals in Eq. (7) cancel. [This cancellation becomes evident if we set $\delta=\infty$ in Eq. (8).] However, for this $\phi^{\infty}$ theory the expectation values $G_{N}$ approach finite limits:

$$
\lim _{\delta \rightarrow \infty} G_{N}=(-i)^{N}
$$

The case $\delta<0$ is more interesting. As $\delta$ decreases below 0 , path 1 rotates clockwise and path 2 rotates anticlockwise. (The paths rotate infinitely fast as $\delta \rightarrow-2$.) Whenever the two paths are horizontal, the resulting $\phi^{2 /(2 k+1)}$ theory is real and there is no symmetry breaking. This happens for the special set of values

$$
\delta=-4 k /(2 k+1) \quad(k=0,1,2,3, \ldots) .
$$

For this case we have

$$
\lim _{\delta \rightarrow-4 k /(2 k+1)} G_{N}=\left\{\begin{array}{cc}
0 & (N \text { odd }), \\
\frac{\Gamma\left[(N+1)\left(\frac{1}{2}+k\right)\right]}{\Gamma\left(\frac{1}{2}+k\right)} & (N \text { even }) .
\end{array}\right.
$$


If the two paths overlap (this occurs when the paths are vertical and go up or down the imaginary axis), there is cancellation. This happens for a $\phi^{1 / k}$ theory when

$$
\delta=(1-2 k) / k \quad(k=0,1,2,3, \ldots) .
$$

For this case we have

$$
\lim _{\delta \rightarrow(1-2 k) / k} G_{N}=(-i)^{N}(-1)^{k N}[k(N+1)] ! / k !,
$$

which reduces to Eq. (17) for the special case $k=0$.

Next, we examine theories in one-dimensional space-time (quantum mechanics). In general, the Hamiltonian

$$
H=p^{2} / 2+(i x)^{2+\delta}
$$

represents a broken-symmetry theory because $H$ is not invariant under the parity operation $\mathcal{P}$, whose effect is to make the replacements $p \rightarrow-p$ and $x \rightarrow-x$. (While $H$ is not symmetric under time reversal $\mathcal{T}$, which makes the replacements $p \rightarrow-p, x \rightarrow x$, and $i \rightarrow-i, H$ is symmetric under $\mathcal{P} \mathcal{T}$.) We emphasize that there are both broken- and unbroken-symmetric phases in zero- and one-dimensional Euclidean space-time, but there is no transition between these different phases. Transitions can only occur when the dimension is two or more.

As in the case of zero dimensions, there is a set of values of $\delta$ [see Eq. (18)] corresponding to quantum-mechanical theories for which there is no broken symmetry. These special theories are described by the Hamiltonians

$$
H=p^{2} / 2-x^{2 /(2 k+1)} \quad(k=0,1,2,3, \ldots),
$$

where we have chosen $i^{2 /(2 k+1)}=-1$. The Hamiltonians in Eq. (23) have a deep connection with quasi-exactly solvable theories in quantum mechanics [13]. To express our theories in a more familiar form we make the change of independent variable $x=t^{2 k+1}$ in the Schrödinger equation $H \psi(x)=E \psi(x)$, followed by the multiplicative change of dependent variable $\psi=t^{k} y$ to eliminate one-derivative terms. We obtain the Schrödinger equation 


$$
y^{\prime \prime}(t)=\left[\left(k^{2}+k\right) t^{-2}-2(2 k+1)^{2} t^{4 k}\left(t^{2}+E\right)\right] y(t) .
$$

Equation (24) represents a particle of zero energy in a rotationally symmetric quasi-exactly solvable potential having a centrifugal barrier.

Finally, we use our new delta-expansion to calculate the one-point Green's function $G_{1}$ in a symmetry-broken scalar quantum field theory in $D$-dimensional Euclidean space. The vacuum-persistence amplitude for the Lagrangian density in Eq. (4)) is

$$
Z=\int \mathcal{D} \phi e^{\int d^{D} x\left[-\frac{1}{2}(\nabla \phi)^{2}-\frac{1}{2} m^{2} \phi^{2}+g(i \phi)^{2+\delta}\right]} .
$$

Since the field $\phi$ has dimensions $M^{(D-2) / 2}$ and the coupling constant $g$ has dimensions $M^{2+\delta-\delta D / 2}$, where $M$ is a mass, we re-express Eq. (25) using dimensionally explicit quantities by substituting $g=\frac{1}{2} M^{2+\delta-\delta D / 2}$. Expanding the exponent to first order in $\delta$ gives

$$
\begin{aligned}
Z= & \int \mathcal{D} \phi e^{-S_{0}-\frac{1}{2} \int d^{D} x\left[\delta M^{2} \phi^{2} \log \left(i \phi M^{1-\frac{D}{2}}\right)+\mathrm{O}\left(\delta^{2}\right)\right]} \\
=\int & \mathcal{D} \phi e^{-S_{0}}\left[1-\frac{1}{2} \delta M^{2} \int d^{D} t \phi^{2}(t)\right. \\
& \left.\times \log \left(i \phi(t) M^{1-\frac{D}{2}}\right)\right]+\mathrm{O}\left(\delta^{2}\right),
\end{aligned}
$$

where $S_{0}=\frac{1}{2} \int d^{D} x\left[(\nabla \phi)^{2}+\mu^{2} \phi^{2}\right]$ and $\mu^{2} \equiv m^{2}+M^{2}$.

To evaluate the one-point Green's function $G_{1}$ we insert a factor of $\phi(0)$ into the integrand of Eq. (26) and divide by $Z$. By symmetry, the resulting expression vanishes at $\delta=0$. To first order in $\delta$ we have

$$
G_{1}=-\delta \frac{\int \mathcal{D} \phi e^{-S_{0}} \phi(0) \int d^{D} t \phi^{2}(t) \log \left(i \phi(t) M^{1-\frac{D}{2}}\right)}{2 M^{-2} \int \mathcal{D} \phi e^{-S_{0}}} .
$$

We now show that $G_{1}$ is pure imaginary: We average the above expression with that obtained by setting $\phi$ to $-\phi$ at every lattice point. Provided that the integrals exist, we obtain the explicitly imaginary result

$$
G_{1}=-i \pi \delta M^{2} \frac{\int \mathcal{D} \phi e^{-S_{0}} \phi(0) \int d^{D} t \phi(t)|\phi(t)|}{4 \int \mathcal{D} \phi e^{-S_{0}}} .
$$

It is not easy to evaluate this functional integral because it contains $|\phi(t)|$, so we employ a limiting process: $\lim _{\alpha \rightarrow 1 / 2} \phi^{2 \alpha}=|\phi|$. Before taking the limit we temporarily treat $\alpha$ as an 
integer. Thus, this limiting process involves continuing off the integers. [We cannot prove the validity of this technique, but we justify this procedure in part by comparing the final result with the first term in Eq. (14) for the case $D=0$.] To evaluate this limit we introduce an external source $J(x)$, and express $G_{1}$ in terms of functional derivatives with respect to $J(x)$ :

$$
\begin{aligned}
G_{1}=- & i \frac{\pi}{4} \delta M^{2} \int d^{D} t \frac{\delta}{\delta J(0)} \\
& \times\left.\lim _{\alpha \rightarrow 1 / 2}\left(\frac{\delta}{\delta J(t)}\right)^{1+2 \alpha} \frac{\mathcal{Z}[J]}{\mathcal{Z}[0]}\right|_{J=0},
\end{aligned}
$$

where

$$
\begin{aligned}
\mathcal{Z}[J] & \equiv \int \mathcal{D} \phi e^{\int d^{D} x\left[-\frac{1}{2}(\nabla \phi)^{2}-\frac{1}{2} \mu^{2} \phi^{2}+J \phi\right]} \\
& =\mathcal{Z}[0] e^{\frac{1}{2} \iint d^{D} x d^{D} y J(x) J(y) \Delta(x, y)},
\end{aligned}
$$

and $\Delta(x, y)$, the free propagator in $D$-dimensional Euclidean space, is the Fourier transform of $1 /\left(p^{2}+\mu^{2}\right)$ :

$$
\begin{aligned}
\Delta(x, y) & =\int \frac{d^{D} p}{(2 \pi)^{D}} e^{-i p \cdot(x-y)} \frac{1}{p^{2}+\mu^{2}} \\
& =\frac{1}{2 \pi}\left(\frac{2 \pi|x-y|}{\mu}\right)^{1-\frac{D}{2}} K_{-1+\frac{D}{2}}(\mu|x-y|) .
\end{aligned}
$$

For integer $\alpha$ we use Eqs. (29) and (30) to evaluate Eq. (28) and obtain the final result for $G_{1}$ :

$$
\begin{aligned}
G_{1}= & -i \frac{\pi}{4} \delta M^{2} \lim _{\alpha \rightarrow 1 / 2} \int d^{D} t \frac{\delta}{\delta J(0)}\left(\frac{\delta}{\delta J(t)}\right)^{1+2 \alpha} \\
& \times\left[\frac{1}{2} \iint d^{D} x d^{D} y J(x) J(y) \Delta(x, y)\right]^{\alpha+1} /(\alpha+1) ! \\
= & -i \frac{\pi}{4} \delta M^{2} \lim _{\alpha \rightarrow 1 / 2} \frac{(2 \alpha+2) ! \Delta^{\alpha}(0,0) \int d^{D} t \Delta(0, t)}{2^{\alpha+1}(\alpha+1) !} \\
= & -i \delta M^{2} \sqrt{\pi \Delta(0,0) / 2} \int d^{D} t \Delta(0, t) \\
= & -i \delta M^{2} \mu^{-3}\left(\mu^{2} / 4 \pi\right)^{D / 4} \sqrt{\pi \Gamma(1-D / 2) / 2},
\end{aligned}
$$

where we have used $\int d^{D} t \Delta(0, t)=\mu^{-2}$ and 


$$
\Delta(0,0)=\mu^{D-2} 2^{-D} \pi^{-D / 2} \Gamma(1-D / 2) \quad(D<2) .
$$

At $D=0, m=0$, and $M=\mu=\sqrt{2}$, Eq. (31) reduces to the first term in Eq. (14). Note that as $D$ increases past $2, G_{1}$ in Eq. (31) becomes real because the gamma function changes sign. For $D<2, \Delta(0,0)$ is finite and positive, but for $D>2$ it is a divergent integral regulated by continuing through complex dimension. Thus, for $D>2$, the sign is a priori unpredictable.

We thank D. Bessis, G. Guralnik, and Z. Guralnik for their invaluable insight. CMB is grateful to the Physics Department at the Technion - Israel Institute of Technology and the Theoretical Physics Group at Imperial College, London, for their hospitality and he thanks the Lady Davis Foundation, the Fulbright Foundation, and the PPARC for financial support. CMB and KAM thank the U.S. Department of Energy for financial support. 


\section{REFERENCES}

* Permanent address: Department of Physics, Washington University, St. Louis, MO 63130, USA.

$\dagger \quad$ E-mail: cmb@howdy.wustl.edu

$\ddagger \quad$ E-mail: kmilton@ou.edu

[1] C. M. Bender, K. A. Milton, S. S. Pinsky, and L. M. Simmons, Jr., J. Math. Phys. 30, 1447 (1989); C. M. Bender, S. Boettcher, and K. A. Milton, J. Math. Phys. 32, 3031 (1991); B. Abraham-Shrauner, C. M. Bender, and R. N. Zitter, J. Math. Phys. 33, 1335 (1992).

[2] C. M. Bender, K. A. Milton, M. Moshe, S. S. Pinsky, and L. M. Simmons, Jr., Phys. Rev. Lett. 58, 2615 (1987); C. M. Bender, K. A. Milton, M. Moshe, S. S. Pinsky, and L. M. Simmons, Jr., Phys. Rev. D 37, 1472 (1988).

[3] C. M. Bender and H. F. Jones, Phys. Rev. D 38, 2526 (1988); H. T. Cho, K. A. Milton, J. Cline, S. S. Pinsky, and L. M. Simmons, Jr., Nucl. Phys. B 329, 574 (1990).

[4] C. M. Bender, K. A. Milton, S. S. Pinsky, and L. M. Simmons, Jr., Phys. Lett. B 205, 493 (1988); C. M. Bender and K. A. Milton, Phys. Rev. D 38, 1310 (1988).

[5] C. M. Bender, F. Cooper, and K. A. Milton, Phys. Rev. D 40, 1354 (1989); C. M. Bender, S. Boettcher, and K. A. Milton, Phys. Rev. D 45, 639 (1992); C. M. Bender, F. Cooper, K. Milton, M. Moshe, S. S. Pinsky, and L. M. Simmons, Jr., Phys. Rev. D 45, 1248 (1992); C. M. Bender, K. A. Milton, and M. Moshe, Phys. Rev. D 45, 1261 (1992).

[6] C. M. Bender, F. Cooper, and K. A. Milton, Phys. Rev. D 39, 3684 (1989); C. M. Bender, F. Cooper, G. Kilkup, P. Roy, and L. M. Simmons, Jr., J. Stat. Phys. 64, 395 (1991). 
[7] C. M. Bender and T. Rebhan, Phys. Rev. D 41, 3269 (1990).

[8] To order $\delta^{2}$ the energy remains real because the imaginary parts in $\left\langle n\left|H_{1}\right| m\right\rangle\left\langle m\left|H_{1}\right| n\right\rangle$ cancel as in Eq. (5). We are convinced that similar cancellations occur to all orders.

[9] Schwinger-Dyson equations, symmetry breaking, and choice of path integral contour have been studied by S. Garcia and G. S. Guralnik, Proceedings of Workshop on Quantum Infrared Physics, American University of Paris (Paris, France, 6-18 June 1994), World Scientific, p. 205; Z. Guralnik, to be published in the Proceedings of Orbis Scientiae 1996.

[10] E. R. Caianello and G. Scarpetta, Nuovo Cimento 22A, 454 (1974); C. M. Bender and R. Z. Roskies, Phys. Rev. D 25, 427 (1982); C. M. Bender and F. Cooper, Phys. Rev. D 39, 2343 (1989).

[11] A simple example is the integral $\int_{0}^{\infty} d t e^{-t / z}$, which exists for $\operatorname{Re} z>0$. When this condition is met, the integral evaluates to $z$, which is an entire function of $z$.

[12] A detailed discussion of the rotation of contours for eigenvalue problems is given in C. M. Bender and A. Turbiner, Phys. Lett. A 173, 442 (1993).

[13] Such potentials are described in A. V. Turbiner, Sov. Phys., J. E. T. P. 67, 230 (1988); M. A. Shifman and A. V. Turbiner, Comm. Math. Phys. 126, 347 (1989); A. G. Ushveridze, Quasi-Exactly Solvable Models in Quantum Mechanics (Institute of Physics, Bristol, 1993) and references therein; A. Krajewska, A. Ushveridze, and Z. Walczak, preprint hep-th/9601088. 